\title{
The Central Logic Board for the optical module of the KM3NeT detector
}

\section{Paolo Musico ${ }^{1}$}

I.N.F.N. Genova

Via Dodecaneso, 3316146 - Genova, Italy

E-mail: Paolo.Musico@ge.infn.it

\section{On behalf of the KM3NeT collaboration}

www.km3net.org

The KM3NeT deep sea neutrino observatory will include a very large number of multiPhotomultiplier (PMT) optical modules (DOM) to detect the Cherenkov light generated by secondary particles produced in neutrino interactions.

The Central Logic Board (CLB) has been developed to acquire timing and amplitude information from the PMT signals, implementing time-to-digital conversion (TDC) with time over threshold (TOT) technique.

The board is also used to configure all the DOM subsystems, to assist in the DOM positioning and orientation, calibration and to monitor temperature and humidity in the DOM itself.

All the collected data are transmitted to shore using a wide-bandwidth optical network.

Moreover, through the optical network, all the DOMs are kept synchronized in time within $1 \mathrm{~ns}$ precision using the White Rabbit (WR) Precision Time Protocol (PTP) over an Ethernet connection.

To implement all these functionalities, a large Field Programmable Gate Array (FPGA) has been adopted.

The logic inside the FPGA is very complex, including two microprocessor systems running concurrent code.

A set of CLB prototypes is now operational, and a batch of 50 pieces has been ordered to equip the first two KM3NeT detection units (DU) which will be deployed during next year.

CLB design considerations, prototyping issues and measurements made on the prototypes will be reported.

Three European institutions are actively working on the design: INFN (Italy), IFIC (Spain), NIKHEF (Netherlands)

Technology and Instrumentation in Particle Physics 2014

2-6 June, 2014

Amsterdam, the Netherlands

${ }^{1}$ Speaker 


\section{Introduction}

The KM3NeT collaboration is designing and building a very large scale $\left(\mathrm{Km}^{3}\right.$ size) deepsea neutrino telescope to be deployed and operated in the Mediterranean Sea. Neutrino-induced charged particles are detected by measuring their Cherenkov light in sea-water, using photomultiplier tubes (PMTs) inside transparent and pressure resistant spherical enclosures. We have successfully developed an optical sensor, the Digital Optical Module (DOM), by placing 31, 3-inch PMTs in a 17-inch glass sphere along with the readout electronics. Thousands of these modules will instrument the sea volume and they will be connected to the shore through electro-optical cables up to $100 \mathrm{Km}$ length.

\section{The Digital Optical Module (DOM)}
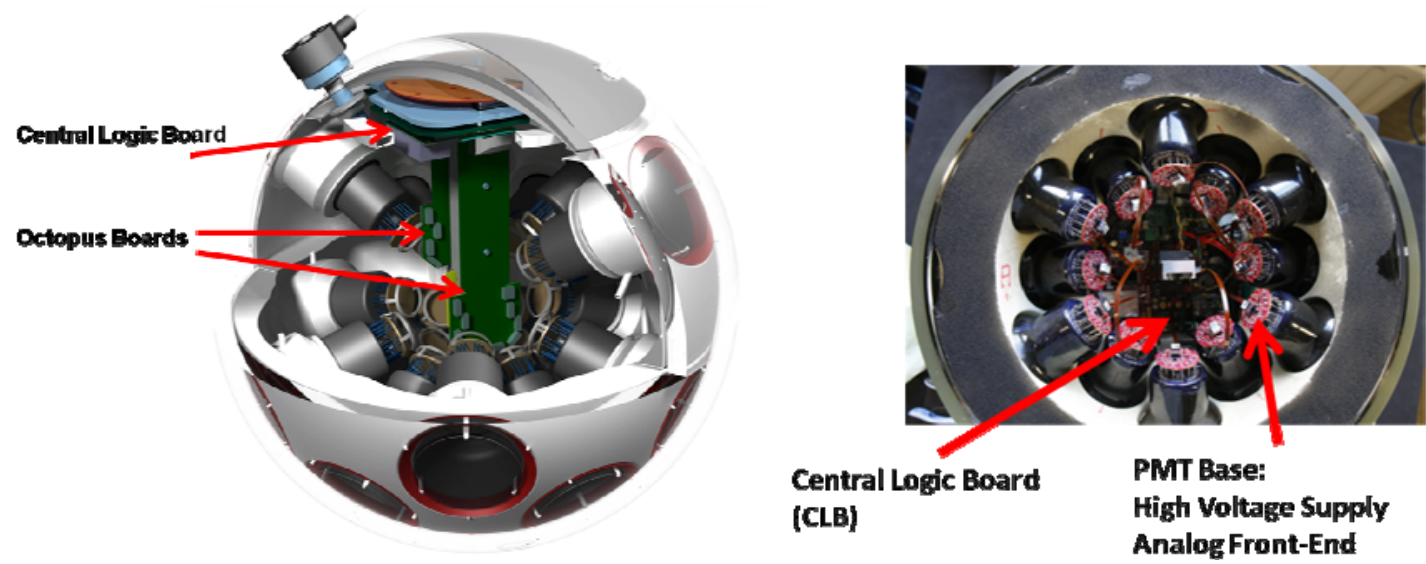

Figure 1: A drawing of the DOM (left) and a picture of one prototype (right)

The DOM is composed by two glass hemispheres. It houses 31 3" PMTs and all the related electronics needed to manage them:

- PMT base board with high voltage bias generator, signal preamplifier and discriminator;

- Octopus boards used to collect signals from PMT bases, configure them and feed the Central Logic Board (CLB);

- Central Logic Board (CLB) used to control the DOM and to handle the communication with the shore station;

- Power Board (underneath the CLB) used to efficiently generate all the needed low voltage power rails. 


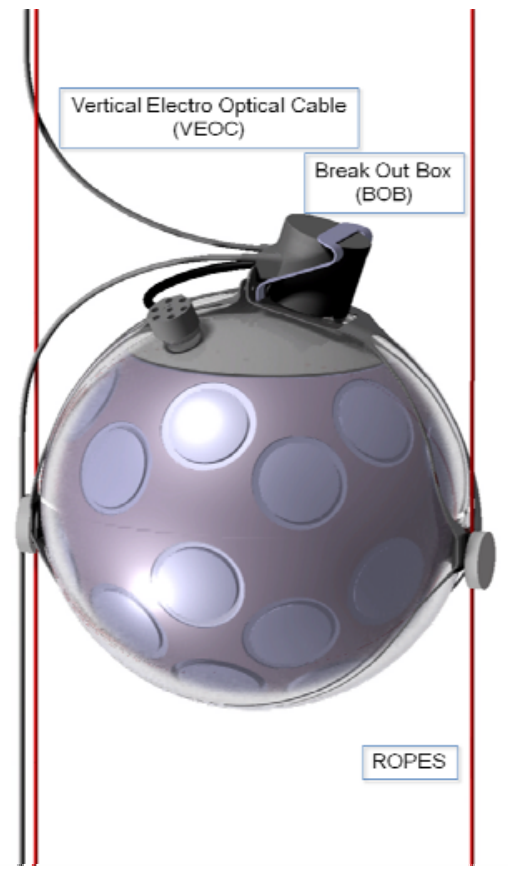

Figure 2: A drawing of the DOM connected to the string structure

Eighteen DOMs will be connected in a vertical string structure called Detection Unit (DU). Only one electro optical cable runs along the string structure carrying the optical fiber for communication and the mid voltage ( $375 \mathrm{~V}$, down converted to $12 \mathrm{~V}$ in the Break Out Box) used to supply all the DOMs in parallel.

\section{The Central Logic Board (CLB)}

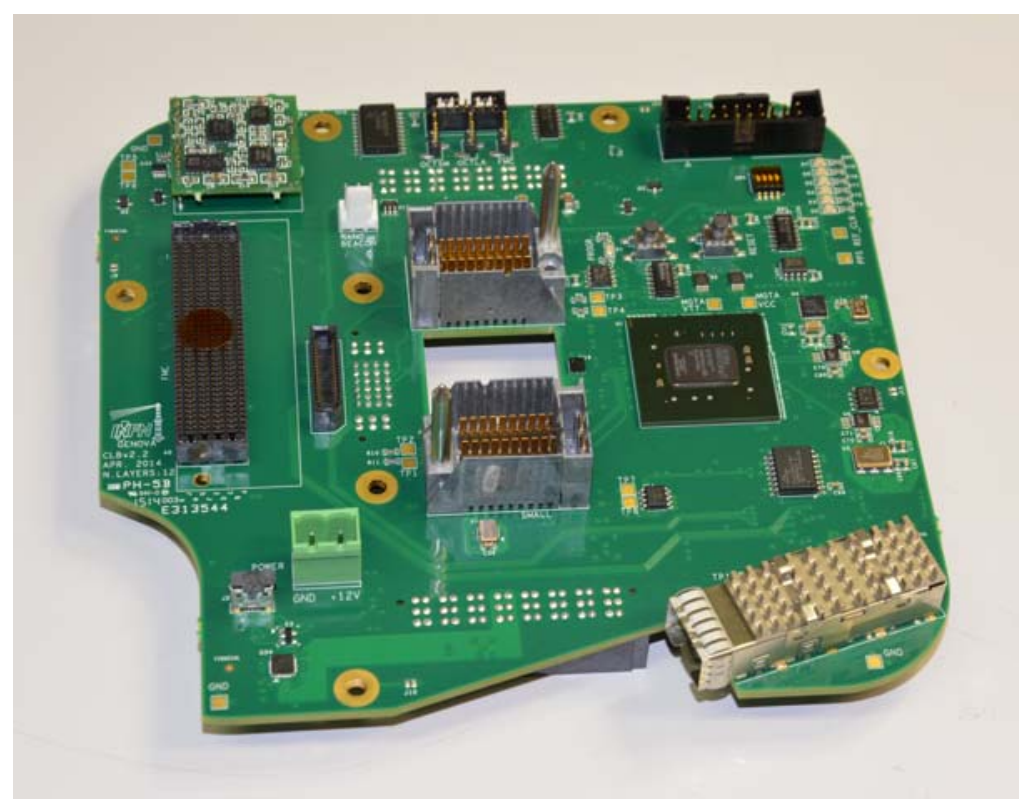

Figure 3: The Central Logic Board (CLB) 
The CLB main components are the following:

- Xilinx Kintex-7 FPGA (XC7K160TBG676) is the core of the board, used to measure the arrival time and the pulse width of the 31 PMT discriminated signals with 1 ns resolution

- Tunable oscillators (20 MHz and $25 \mathrm{MHz}$ base frequencies) for White Rabbit PTP core

- 512 Mbit Quad SPI Flash Eprom

- PMT interface (31 x) through Octopus boards connectors

- SFP laser module housing with heatsink

- Temperature \& Humidity sensor

- Compass \& Tilt meter

- NanoBeacon (calibration LED Flasher) Interface

- Acoustic AES interfaces for Hydrophone and Piezoelectric acoustic sensors

- Expansion industry standard FMC High Pin Count connector

- Debugging: Serial to USB converter (2 x), Standard Header 20 pin connector, High density connector for batch production testing, Dip-Switches \& LEDS

CLB power consumption: $<4.5 \mathrm{~W}$ Total DOM power consumption will be about $7.5 \mathrm{~W}$

In the FPGA two microprocessor systems based on LM32 soft processor are implemented:

- White Rabbit Precision Time Protocol engine: implement 1ns time synchronization and transfer data to shore station;

- Configuration and slow control: handles all the devices inside the DOM, manage housekeeping data and implement debug ports.

\subsection{Building and testing}

The first batch of 8 prototypes have been built by mid December 2013 and tested in the first months of 2014.

A second batch of 12 prototypes (with some marginal modifications) have been delivered at the end of April 2014 and extensive tests are in progress.

Preserie production of 50 pieces is in progress and a tender for the 600 pieces batch production has been assigned.

The first test bench for the whole DOM is under preparation at NIKHEF. It includes a whole set of PMT (with bases), two Octopus boards, one CLB, one Power Board.

WR PTP has been tested with $200 \mathrm{Mbit} / \mathrm{s}$ Ethernet traffic originated in the CLB and connected to a dedicated switch together other data generators.

The CLB will be used also as controller in the Base Container placed on the sea floor at the bottom of the DU. It will handle all the power control switch and the optical components needed to operate the DU. In this case a custom FMC mezzanine will be used to interface the various parts of the DU base. 


\section{References}

[1] D. Real, Digital Optical Module Read-Out Electronics System of the KM3NeT Neutrino Telescope, in proceedings TIPP2014.

[2] D. Calvo, Nanobeacon and Laser Beacon: KM3NeT Time Calibration Devices, in proceedings TIPP2014.

[3] K. Manolopoulos, Design and development of the Power Supply Board within the Digital Optical Module in KM3NeT, in proceedings TIPP2014.

[4] M. Mendez, KM3NeT On-shore Station and Broadcast Customization of White-Rabbit Switches Towards Optimizing Communication Resources for Shared Control Link, in proceedings TIPP2014. 